\title{
Fecal microbiota transplantation for multiple organ dysfunction syndrome
}

\author{
Nathan J. Klingensmith ${ }^{1}$ and Craig M. Coopersmith ${ }^{1,2^{*}}$ \\ See related research by Wei et al. https://ccforum.biomedcentral.com/articles/10.1186/s13054-016-1491-2
}

Keywords: Sepsis, Fecal microbial transplantation, MODS, Diarrhea, Therapeutic efficacy, 16s rRNA, Firmicutes, Proteobacteria

Approximately 40 trillion bacteria reside inside the human intestine, meaning there are at least as many cells of microbial origin as human origin [1]. While it was once believed that bacteria and humans simply coexisted in the same space, a wide body of evidence now suggests that host-microbial communication is more complex than ever imagined and the microbiome plays a critical role in maintaining host homeostasis. The microbiome is also altered in multiple disease states, including heart disease [2], cancer [3], and Clostridium difficile infection [4], with changes detectable in microbial composition, number, diversity, and virulence compared to healthy controls. While the majority of studies linking the microbiome to disease are associative, there is increasing evidence that the microbiome plays a crucial role in mediating the pathophysiology of multiple acute and chronic illnesses.

The gut has long been hypothesized to be "the motor" of multiple organ dysfunction syndrome (MODS) [5]. Notably, the microbiome is markedly altered in the intensive care unit (ICU). A recent study sampling stool from 115 critically ill patients revealed decreases in Firmicutes and Bacteroidetes-bacteria that are prevalent in the healthy intestine-with increases in opportunistic Proteobacteria [6]. Although these findings cannot determine whether an altered microbiome functions as a marker or mediator in the development of MODS, it raises the question as to whether altering microbial ecology and restoring diversity of the microbiome may be a novel therapeutic strategy in critical illness.

\footnotetext{
* Correspondence: cmcoop3@emory.edu

${ }^{1}$ Department of Surgery and Emory Critical Care Center, Emory University School of Medicine, Atlanta, GA, USA

2Emory University School of Medicine, 101 Woodruff Circle, Suite WMB 5105, Atlanta, GA 30322, USA
}

Multiple methods to manipulate host bacteria have been tried in patients, ranging from giving exogenous bacteria in the form of probiotics to selecting out pathogenic microbes via selective decontamination of the digestive tract. While each of these strategies has shown some clinical promise $[7,8]$, debatably the most effective method of altering the microbiome in human disease is fecal microbial transplantation (FMT). FMT is a procedure where stool is collected from a healthy donor, filtered for particulate matter, and the liquid portion given to the patient via nasogastric tube or via the rectum [9]. TT has been demonstrated to be significantly more efin in recurrent $C$. difficile infections than other able therapies [10]. Treatment failures still occur, ever, which is not surprising given that we do not fully understand the mechanisms responsible for the of FMT, nor do we know the optimal dose and FMT and impact outcomes [11, 12].

The literature on FMT in critical care is limited to a single case report [11]. The barriers to utilizing FMT in the ICU are significant. The impact of giving bacteria to a patient with a markedly altered microbiome who likely has a component of immunosuppression is unknown and has inherent theoretical risks. In addition, the majority of itically ill patients receive antibiotics at some point during their ICU stay, and initiation or continuation of antibiotic therapy would be expected to markedly alter the microbiome following FMT. As such, there must be a commitment to stopping antibiotics both prior to and following FMT to allow the transplanted bacteria to take hold and remodel the microbiome. This is a difficult 
action to take unless it is clear that a patient is not infected, a clinical judgment which unfortunately is not always straightforward at the bedside.

Wei et al. recently reported the use of FMT in two critically ill patients who developed sepsis during their hospital course [13]. Each was successfully treated with multiple antibiotics (which sterilized their cultures) but was left with MODS and non-C. difficile diarrhea, refractory to standard medical management. Analysis of stool from both patients demonstrated marked alterations in the microbiome compared to healthy patients. The investigators concluded that the patients suffered from intestinal dysbiosis not related to active infection and would therefore be appropriate candidates for FMT. Antibiotics were held, and three days later FMT was performed. Over the 2-3 weeks following FMT, both patients had resolution of their diarrhea and improvement in multiple markers of inflammation such as interleukin-6, $C$ reactive protein, procalcitonin, and erythrocyte sedimentation rate. Notably, the investigators followed the bacterial composition of the patients' stool using 16s rRNA pyrosequencing, both immediately before and up to 20 days after FMT. Following FMT, the composition and diversity in both patients shifted toward that of the donor with increasing Firmicutes and decreasing Proteobacteria.

Outside of antibiotics, supportive care remains the mainstay of treatment in the ICU [14], and any new therapy that has the capacity to cure a condition in critical illness is potentially exciting. However, the efficacy seen with FMT in this study must be interpreted with caution. First, while a rich and diverse intestinal microbiome is associated with health [15], this does not inherently mean that the altered microbiome plays a causative role in MODS or that restoration to a normal state in critical illness is beneficial. In the cases presented here, stool volume and inflammatory markers were already decreasing prior to initiation of FMT, and it is possible the patients would have recovered without receiving this experimental therapy. Assuming FMT was indeed responsible for the ultimate improvement in patient course, the degree of generalizability of the results presented is unclear, as with any case report. For instance, if one imagines a clinical trial of FMT that mimics the patients studied, the entry criteria would be relatively narrow-patients with MODS and dysbiosis-induced non-infectious diarrhea who do not require antibiotics. As such, FMT must be considered experimental in critical illness until rigorous trials are conducted. Nonetheless, the opportunity to manipulate our microbiome for therapeutic benefit in the ICU represents a tantalizing new direction in the future care of critically ill patients.
Funding

This work was supported by funding from the National Institutes of Health (GM072808, GM095442, GM104323, GM109779, GM113228).

\section{Authors' contributions}

NK and CC drafted the manuscript and reviewed the final version of the text. Both authors read and approved the final manuscript.

\section{Competing interests}

The authors declare that they have no competing interests.

\section{Consent for publication}

Not applicable.

Published online: 19 December 2016

\section{References}

1. Sender R, Fuchs $S$, Milo R. Are we really vastly outnumbered? Revisiting the ratio of bacterial to host cells in humans. Cell. 2016;164(3):337-40.

2. Kelly TN, Bazzano LA, Ajami NJ, He H, Zhao J, Petrosino JF, et al. Gut microbiome associates with lifetime cardiovascular disease risk profile among bogalusa heart study participants. Circ Res. 2016;119(8):956-64.

3. Rajagopala SV, Yooseph S, Harkins DM, Moncera KJ, Zabokrtsky KB, Torralba MG, et al. Gastrointestinal microbial populations can distinguish pediatric and adolescent acute lymphoblastic leukemia (ALL) at the time of disease diagnosis. BMC Genomics. 2016;17(1):635.

4. Antharam VC, Li EC, Ishmael A, Sharma A, Mai V, Rand KH, et al. Intestinal dysbiosis and depletion of butyrogenic bacteria in Clostridium difficile infection and nosocomial diarrhea. J Clin Microbiol. 2013;51(9):2884-92.

5. Klingensmith NJ, Coopersmith CM. The gut as the motor of multiple organ dysfunction in critical illness. Crit Care Clin. 2016;32(2):203-12.

6. McDonald D, Ackermann G, Khailova L, Baird C, Heyland D, Kozar R, et al. Extreme dysbiosis of the microbiome in critical illness. mSphere. 2016;1(4): e00199-16.

7. Price R, MacLennan G, Glen J. Selective digestive or oropharyngeal decontamination and topical oropharyngeal chlorhexidine for prevention of death in general intensive care: systematic review and network metaanalysis. BMJ. 2014;348:g2197. http://www.ncbi.nlm.nih.gov/pmc/articles/ PMC3970764/.

8. Manzanares W, Lemieux M, Langlois PL, Wischmeyer PE. Probiotic and synbiotic therapy in critical illness: a systematic review and meta-analysis. Crit Care. 2016;19:262. http://www.ncbi.n/m.nih.gov/pmc/articles/ PMC4991010/.

9. Perez $\mathrm{E}$, Lee $\mathrm{CH}$, Petrof EO. A practical method for preparation of fecal microbiota transplantation. Methods Mol Biol Clifton NJ. 2016;1476:259-67.

10. Chapman BC, Moore HB, Overbey DM, Morton AP, Harnke B, Gerich ME, et al. Fecal microbiota transplant in patients with Clostridium difficile infection: a systematic review. J Trauma Acute Care Surg. 2016;81(4):756-64.

11. Li Q, Wang C, Tang C, He Q, Zhao X, Li N, et al. Successful treatment of severe sepsis and diarrhea after vagotomy utilizing fecal microbiota transplantation: a case report. Crit Care. 2015;19:37. http://www.ncbi.nlm.nih. gov/pmc/articles/PMC4346118/.

12. Bojanova DP, Bordenstein SR. Fecal transplants: what is being transferred? PLoS Biol. 2016;14(7), e1002503.

13. Wei Y, Yang J, Wang J, Yang Y, Huang J, Gong H, et al. Successful treatment with fecal microbiota tranplantation in patients with multiple organ dysfunction syndrome and diarrhea following severe sepsis. Crit Care. 2016; 20(1):332.

14. Dellinger RP, Levy MM, Rhodes A, Annane D, Gerlach H, Opal SM, et al. Surviving sepsis campaign: international guidelines for management of severe sepsis and septic shock: 2012. Crit Care Med. 2013;41(2):580-637.

15. Suau A, Bonnet R, Sutren M, Godon J-J, Gibson GR, Collins MD, et al. Direct analysis of genes encoding $16 \mathrm{~S}$ rRNA from complex communities reveals many novel molecular species within the human gut. Appl Environ Microbiol. 1999:65(11):4799.

\section{Abbreviations}

FMT: Fecal microbial transplantation; ICU: intensive care unit; MODS: multiple organ dysfunction syndrome. 